\title{
Meta-analysis of the effects of smoking prevention programs for young adolescents
}

\author{
Rhayun Song ${ }^{1}$, Moonkyoung Park ${ }^{2}$ \\ ${ }^{1}$ Professor, College of Nursing, Chungnam National University, Daejeon; ${ }^{2}$ Assistant Professor, College of Nursing, Chungnam National University, Daejeon, Korea
}

\begin{abstract}
Purpose: This meta-analysis aimed to analyze the effects of smoking prevention programs for young adolescents at early smoking stages to identify the appropriate characteristics of prevention programs for this population. Methods: Searches of health-related databases and Google Scholar were conducted, and 23 randomized studies were included in the analysis. The main outcome variable was smoking behavior. The analysis was conducted using Comprehensive Meta-Analysis software (version 3.0). Results: Smoking prevention programs significantly reduced smoking behaviors ( $\mathrm{OR}=0.85,95 \% \mathrm{CI}=0.77-0.93$ ). School-based programs $(\mathrm{OR}=0.79,95 \% \mathrm{CI}=0.75-0.83)$, programs by trained teachers or educators (OR=0.77, 95\% CI=0.71-0.83), high-intensity programs (OR=0.82, 95\% CI=0.75 $-0.91)$, and programs in an in-school setting ( $\mathrm{OR}=0.82,95 \% \mathrm{CI}=0.74-0.90)$ had the most significant effect on reducing smoking behavior. Conclusion: For young adolescents, smoking prevention programs are most effective when they are school-based or highintensity programs, and when conducted by teachers or educators with proper training. Further studies are required since there was insufficient research to explore the effect of web-based programs or family-centered programs on adolescent smokers.
\end{abstract}

Key words: Smoking prevention; Meta-analysis; Adolescent; Health behavior
Corresponding author

Moonkyoung Park

College of Nursing, Chungnam National University, 266, Munhwaro, Jung-gu,

Daejeon 35015, Korea

TEL: +82-42-580-8325

FAX: $+82-42-580-8309$

E-MAIL: lunarnr@cnu.ac.kr

Received Feb 2, 2021

Revised Mar 15, 2021

Accepted Mar 24, 2021

\section{INTRODUCTION}

\section{Need for Study}

Most smokers begin to smoke in their adolescent years [1]. Initiation of smoking during adolescence may cause cardio-cerebrovascular diseases such as hypertension or dyslipidemia [2], certain types of cancer [1], psychological problems including depression and suicide, and serious behavioral problems within the family or at school [2]. Diseases related to smoking are responsible for premature death or poor quality of life for approximately 6.4 million adults every year [3]. This is the main reason health care professionals recommend strict policies to discourage adolescents from smoking. Due to various policy measures in South Korea, the rate of smoking among adolescents showed a decreasing trend until 2016. However, the adolescent smoking rate increased to $6.7 \%$ in 2019. In addition, the mean age at which adolescents tried their first cigarette dropped to 12.7 years old. This particular age corresponds to middle school, for which the current smok- ing rate is $1 \%$ among first-year students, with an increase to $5 \%$ among third-year students. Approximately $20 \%$ of adolescents with smoking experience smoke daily year-round [4]. Most adolescents who smoke fail to quit smoking even when they want to quit [1].

Since the frontal lobe of the brain is still developing, adolescents are more vulnerable to impulsive and dangerous actions and temptations [5]. Adolescents tend to initiate smoking without hesitation since they underestimate its risks and often feel overconfident about their health [6]. Unlike adulthood, adolescence is a period during which individuals are more vulnerable to, and can become more dependent on, addictive substances. Individuals who start smoking during adolescence are likely to continue smoking during adulthood as well [7]. Therefore, the implementation of effective smoking prevention programs should be targeted to those entering middle school, when adolescents are most likely to begin smoking.

Smoking cessation programs, which include various strategies such as life skills training [8], motivation enhancement [9], or cognitive behavioral approaches [10], have been con- 
ducted to improve knowledge, attitude, and coping skills in adolescents. These programs are designed to increase adolescents' willingness to quit smoking. However, the effectiveness of these programs showed mixed results on controlling smoking-related behaviors [11]. A meta-analysis study on the effect of one smoking prevention program among female students aged 18 and younger showed that the program's effectiveness at smoking prevention was not certain [12]. A systematic review and meta-analysis of randomized and nonrandomized studies found that school or local communitybased programs improved knowledge and attitude toward smoking behaviors among adolescents and young adults, but had an insignificant effect on smoking behaviors [13]. A study analyzing the effects of web-based smoking prevention programs showed they were effective in the short-term at stopping smoking behaviors, increasing adolescents' willingness to quit smoking, and changing attitudes toward smoking, but the long-term effects were insignificant [14]. This suggests that the effectiveness of smoking prevention programs for adolescents may differ depending on their duration. Smoking prevention programs also tend to be conducted for a wide age range, from children to late adolescents, without considering the unique developmental characteristics of young adolescents.

A program's intensity is another point to consider to understand the effects of smoking prevention programs. In a metaanalysis, Sussman, Sun, and Dent [15] observed no difference in the smoking rate when smoking prevention programs were implemented five or more times. Kim et al. [10] meta-analyzed the effectiveness of smoking prevention programs among adolescents in South Korea. The results showed that the average intensity of the interventions was 6.5 sessions, but a high intensity level had only a small effect on self-efficacy and willingness to quit smoking and was not effective at reducing smoking. Moreover, the participants consisted of middle school and high school students, so the findings do not necessarily apply when considering the effect of intervention intensity on young adolescents.

Providers of smoking prevention programs and their methods varied among studies. Smoking prevention programs led by medical school students [16], school nurses [17], and trained education providers showed mixed results regarding their effectiveness $[18,19]$. This suggests that the effectiveness of smoking prevention programs may be influenced by the program provider.

Previous systematic reviews or meta-analyses mainly focused on smoking prevention either among adults or across the entire childhood-adolescence period. Early intervention is crucial, as smoking among adolescents typically begins early, and the rate of adolescent smoking significantly increases with grade level. Therefore, this study aimed to identify the most effective features of smoking prevention programs at reducing smoking behavior among young adolescents.

A meta-analysis was conducted by selecting studies in which smoking prevention programs were conducted for young adolescents in randomized controlled trials (RCTs), and which studied the effects of programs on smoking behavior according to the program characteristics. This meta-analysis was undertaken to provide objective evidence for selecting, designing, and conducting smoking prevention programs that are appropriate for the developmental characteristics of young adolescents.

\section{Purpose}

A systematic review and meta-analysis of RCTs was conducted to identify the effect of smoking prevention programs on the initiation and continuation of smoking behavior among young adolescents. The specific objectives of this study were as follows:

- To identify the general characteristics of smoking prevention programs

- To determine the total effect size and short- and longterm effects of smoking prevention programs on smoking behavior among young adolescents

- To analyze the effect of smoking prevention programs on smoking behavior according to the general characteristics (program type, provider, setting, and intensity) of prevention programs

\section{METHODS}

Ethics statement: This study is a literature review of previously published studies and was therefore exempt from institutional review board approval.

\section{Study Design}

This study is a systematic review and meta-analysis aiming to objectively identify the effects of smoking prevention programs for young adolescents.

\section{Inclusion and Exclusion Criteria of Data}

The selection process of studies followed the Preferred Reporting Items for Systematic Reviews and Meta-analyses (PRISMA) [20]. The detailed inclusion criteria are as follows:

\section{1) Participants}

The definition of early adolescence varies somewhat from 
study to study. The criteria for participants in this study were taken from a previous study [21] that examined young adolescents between the ages of 10 and 14 who were in the fourth grade of elementary school to the second grade of middle school. Studies that included pregnant adolescents or adolescents with psychological diseases, emotional and behavioral problems, and developmental disorders were excluded.

\section{2) Interventions}

Smoking prevention programs were defined as nonpharmacologic interventions designed to prevent or end smoking behavior. In this study, any intervention program with a main goal of preventing or ending smoking behavior was included. In addition, intervention programs for substance abuse or cancer prevention that included an educational component on prevention or cessation of smoking were also considered smoking prevention programs. No limitation was placed on the application period or environment of the program. However, studies that incorporated smoking cessation products such as nicotine gum or patches, policies requiring smoking cessation, second-hand smoking prevention intervention, or exercise intervention were excluded.

\section{3) Comparisons}

The comparative group consisted of no treatment or usual care.

\section{4) Outcomes}

Possible outcomes in terms of smoking behavior were initiation, continuation (smoking experience within the past month), or cessation of smoking. The number of subjects or the ratio for these factors after participating in a smoking prevention program was used.

\section{5) Study design}

RCTs with a control group were selected in order to establish an evidence base with a high level of certainty.

\section{Data Search and Adoption Process}

As a meta-analysis of previous research, this study received an Institutional Review Board (IRB) review exemption (No. 201810-SB-160-01) from the institution to which the researchers were affiliated. The period in which the search was conducted was from September 20 to October 31, 2018. The search parameters included studies published in Korean or English since 2000 to include only relatively recent interventions. In addition to published papers, theses and reports were also included in the search to minimize publication bias. When published papers and theses overlapped, the published work was selected. When subjects overlapped, the work that was published earlier was selected. Searches were conducted independently by two researchers using international databases such as PubMed, CINAHL, ProQuest, and Cochrane Library, and Korean databases such as KISS, RISS, and DBpia. References in studies were also examined. A manual search in Google Scholar was also conducted.

Medical subject headings (MeSH) terms and related synonyms were searched with Boolean operators and truncation to increase the sensitivity. The search formula for English-language databases included ("adolescent" [MeSH] OR "youth" [tiab] OR "child*" [tiab] OR "adolescen" [tiab] OR "school age" [tiab] OR "secondary school" [tiab] OR "young" [tiab] OR "girl" [tiab] OR "boy*" [tiab]) AND ("smoking cessation" [MeSH] OR "smoking" [MeSH] OR "smoking prevention" [MeSH] OR "abuse" [tiab] OR "smoke" [tiab] OR "tobacco" [tiab] OR "cigarette" [tiab] OR "substance abuse" [tiab] OR "drug abuse" [tiab]) AND ("randomized controlled trials as topic" [MeSH] OR "random*" [tiab]). The same combination of search terms was translated into Korean and used for the Korean-language databases. The standard search range specified in the Core, Standard, and Ideal (COSI) model proposed by the U.S. National Library of Medicine was used. However, this search strategy did not yield a preponderance of recent results, as a vast number of accumulated studies on smoking prevention interventions have been published and were included in the search. A further complication is that the effects of some interventions were reported as both positive and negative in different studies.

EndNote X9.3 was used to sort and remove duplicate studies from the searches of online databases. Researchers verified whether the search words were included in the title and abstract of studies that met the inclusion criteria. The final selection of studies was based on the full text.

\section{Data Analysis}

\section{1) Quality assessment of studies subject to analysis and publi-} cation bias verification

The quality assessment of the studies was based on the Scottish Intercollegiate Guidelines Network (SIGN) checklist for RCTs [22], as it enables the most comprehensive evaluation of RCT studies. The checklist consists of 10 items, which include: a clearly focused question, random assignment, a concealment method, double-blinding, homogeneity of subjects, the treatment as the only difference, the usage of a tool that has been verified for validity and reliability, withdrawal rate, intention-to-treat (ITT), and reporting the results from all sites. One additional item was applied to evaluate the risk of bias. Two researchers with extensive experience in conduct- 
ing meta-analyses independently conducted the quality assessment and verification of results. The final results were agreed on through discussion if the initial results did not match.

Publication bias is caused by failure to collect search results for unpublished works, resulting in an analysis based solely on published works. In order to assess the presence of publication bias, a funnel plot was used to visualize the distribution of the effect size. If the distribution was even with the vertical line in the center of the plot, the results were considered to have no publication bias [23]. In addition, the Egger regression test [24] was used, at the significance level of $p>$ .050 in the case of uneven distribution. If publication bias was found, the effect size was adjusted using the trim-and-fill method [24] and compared with the results of the original effect size to confirm whether consistency was maintained.

\section{2) Statistical meta-analysis process}

The Comprehensive Meta-Analysis (CMA) version 3.0 program was used in this study for integration of the effect size, homogeneity, and publication bias verification. The effect size was computed based on the odds ratio (OR) using the ratio of the initiation, continuation, or cessation of smoking, or the frequency of occurrence. The significance of the effect size was verified based on a 95\% confidence interval (CI) and the Z-test $(p<.050)$. The Higgin $\mathrm{I}^{2}$ statistic was used to test for the homogeneity of the effect size $\left(\mathrm{I}^{2}<50 \%\right)$. The effect size was calculated based on a random-effect model since the included studies were not all from the same population and observed varied age ranges, though they were all still limited to early adolescence [25]. The mean effect size was used when two or more outcome variables were reported in one study [23].

Sub-group analyses were performed according to the shortterm and long-term effects of the program, program type, program provider, program setting, and program intensity. The short-term effects of a program were defined as a program's outcomes within 1 year of it being conducted. The long-term effects were considered a program's outcomes lasting more than 1 year after a program has been conducted. Programs were considered school-based if they were conducted in schools, family-based if they were conducted among families, or web-based if they were provided on a computer or online. Program providers consisted of trained teachers, health-related persons (school nurse, medical school student, or college student majoring in psychology), or others (web-based or by a peer). Program setting was also classified as either in-school only or other (a combination of inside and outside school, outside school, or web-based). Program intensity was measured according to the criteria used in a study by Kim et al. [10]. Programs were either considered high-intensity (seven or more sessions conducted) or low-intensity (fewer than seven sessions conducted).

\section{RESULTS}

\section{Literature Inclusion Process and Quality Assessment Results}

Literature was included in the meta-analysis according to the PRISMA 2009 flow diagram [20] (Figure 1). Using the established combination of search terms, a total of 7,860 studies were identified from international and South Korean online databases. Additionally, a manual search was conducted for references and on Google Scholar to find gray literature. Overall, 20,502 search results were obtained. Overlapping studies were excluded after reviewing the titles and abstracts of studies found in international and South Korean databases. A total of 260 studies were selected to be screened for inclusion and exclusion criteria in terms of subjects, the research design, the intervention, and outcome variables. After reviewing the full text, studies were excluded for the following reasons: (1) the participants were not young adolescents, (2) the data were not available for meta-analysis, (3) the study analyzed the effects of second-hand smoking prevention programs or advertisements, and (4) the results were analyzed at a school level rather than at an individual level. Two researchers with extensive experience in meta-analyses independently performed content analysis and quality assessment for the selected literature according to the participant, intervention, comparison, outcome, and study design (PICO-SD) process [23], and 24 studies were considered appropriate for analysis. Two studies had overlapping subjects with the results measured at different times, and were considered too similar. As a result, 23 studies were included in the final analysis.

When quality assessment was performed using the SIGN checklist [22], the research topic and purpose were clearly specified in all included studies. Randomized assignment was performed and random numbers were generated to assign experiment and control groups at the school level in all studies. Blinding was deemed to have been properly applied since program providers were not included in data collection in all studies. The withdrawal rate of subjects was reported to be less than 33\%. Moreover, each study involved at least 292 subjects. Therefore, the number of subjects included in the studies was sufficiently large. A total of eight studies were given an ITT analysis in which all participants were analyzed as the initial group (Table 1). The effect size was not analyzed according to the quality level of the literature since the quality of the selected studies was considered outstanding. 


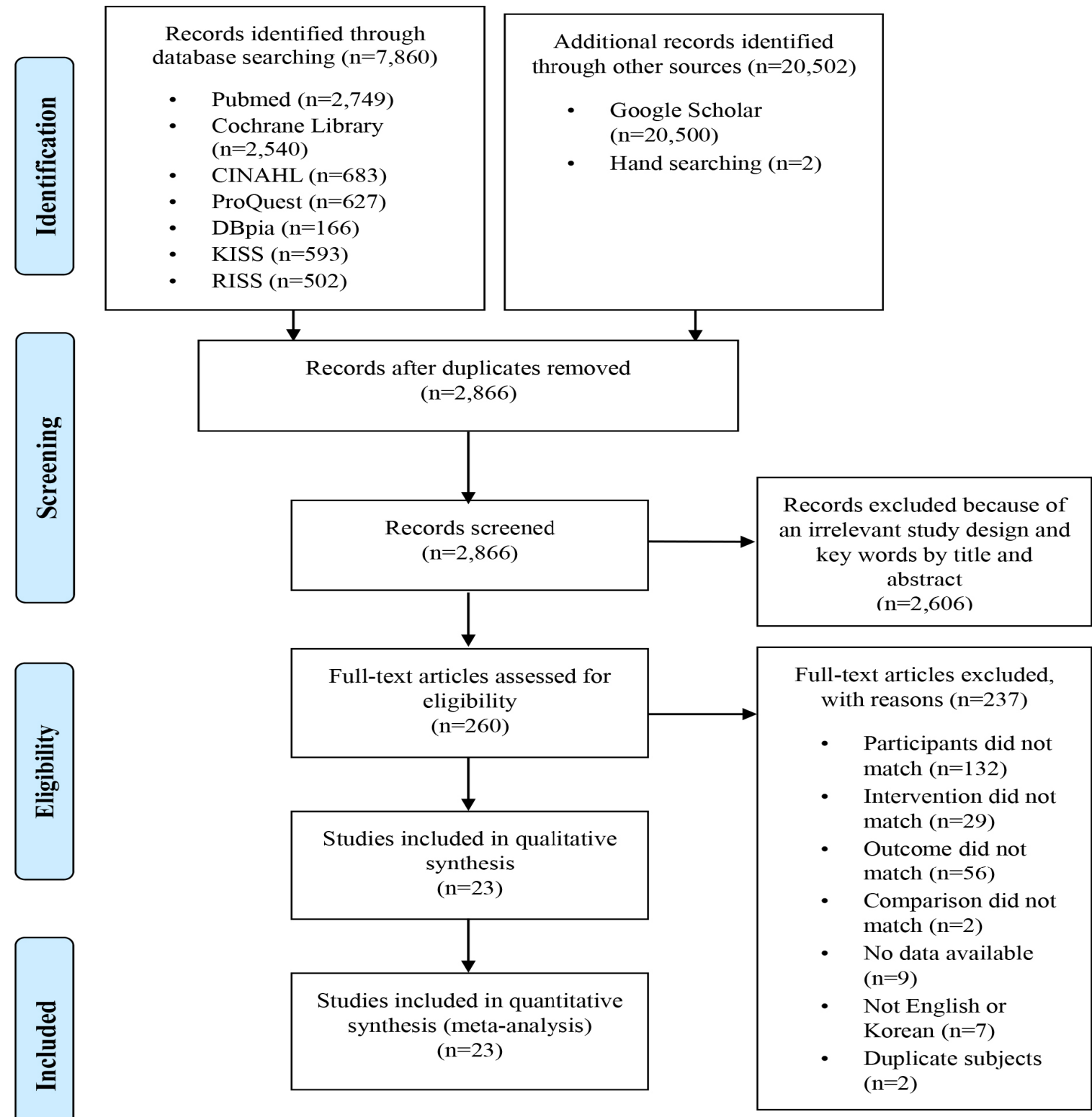

Figure 1. Flow diagram for study selection process.

\section{General Characteristics of the Included Studies}

Among the 23 studies included in the analysis, 14 studies observed smoking prevention programs, while nine studies observed combined programs for smoking and substance abuse prevention. Most studies were conducted in the United States $(k=5)$, the Netherlands $(k=5)$, and Germany $(k=4)$, followed by Spain $(k=2)$. Eighteen studies observed school-based programs, three observed family-centered programs, and two observed web-based programs. Fifteen studies included programs conducted in school settings, while eight studies included programs conducted in other settings (Table 2).
The theoretical background of each program was described in 21 studies, with social influence theory being used primarily in eight studies. There were four studies with unclear theoretical backgrounds. Nine of the studies proposed two or more theoretical backgrounds or used reconstructed models based on more than two theories. Most intervention providers were trained teachers or educators (in 19 studies), but others included a school nurse, a medical school student, a college student majoring in psychology, and a peer (Table 2).

The main target components of the programs were smoking prevention $(\mathrm{k}=13)$, substance abuse prevention including smoking $(\mathrm{k}=9)$, and smoking and alcohol prevention $(\mathrm{k}=1)$ (Table 2). 
Table 1. Quality Assessment of the Included Studies $(N=23)$

\begin{tabular}{|c|c|c|c|c|c|c|}
\hline Author (year) & $\begin{array}{l}\text { Focused } \\
\text { question }\end{array}$ & $\begin{array}{c}\text { Random } \\
\text { assignment }\end{array}$ & $\begin{array}{c}\text { Allocation } \\
\text { concealment }\end{array}$ & Blindness & $\begin{array}{l}\text { Similar at } \\
\text { baseline }\end{array}$ & $\begin{array}{l}\text { Intention to treat } \\
\text { analysis }\end{array}$ \\
\hline Bauman (2001) & Yes & Yes & Yes & Yes & Yes & No \\
\hline Ausems (2002) & Yes & Yes & Yes & Yes & Yes & No \\
\hline Crone (2003) & Yes & Yes & Yes & Yes & Yes & No \\
\hline Curry (2003) & Yes & Yes & Yes & Yes & Yes & Yes \\
\hline Ellickson (2003) & Yes & Yes & Yes & Yes & Yes & No \\
\hline Ausems (2004) & Yes & Yes & Yes & Yes & Yes & No \\
\hline Chou (2006) & Yes & Yes & Yes & Yes & Yes & No \\
\hline Spoth (2007) & Yes & Yes & Yes & Yes & Yes & Yes \\
\hline Vartiainen (2007) & Yes & Yes & Yes & Yes & Yes & No \\
\hline Campbell (2008) & Yes & Yes & Yes & Yes & Yes & No \\
\hline Faggiano $(2008,2010)$ & Yes & Yes & Yes & Yes & Yes & Yes \\
\hline Ringwalt (2010) & Yes & Yes & Yes & Yes & Yes & No \\
\hline Gabrhelik (2012) & Yes & Yes & Yes & Yes & Yes & No \\
\hline Menrath (2012) & Yes & Yes & Yes & Yes & Yes & Yes \\
\hline Luna-Adame (2013) & Yes & Yes & Yes & Yes & Yes & No \\
\hline Malmberg (2014) & Yes & Yes & Yes & Yes & Yes & Yes \\
\hline Andersen (2015) & Yes & Yes & Yes & Yes & Yes & Yes \\
\hline Cremers (2015) & Yes & Yes & Yes & Yes & Yes & No \\
\hline Guo (2015) & Yes & Yes & Yes & Yes & Yes & No \\
\hline Valdivieso Lopez (2015) & Yes & Yes & Yes & Yes & Yes & No \\
\hline Baldus (2016) & Yes & Yes & Yes & Yes & Yes & Yes \\
\hline Krist (2016) & Yes & Yes & Yes & Yes & Yes & Yes \\
\hline Brinker (2017) & Yes & Yes & Yes & Yes & Yes & No \\
\hline
\end{tabular}

The intensity and content of programs varied across the included studies. In one study, a family-centered program was conducted intermittently for a short period of time through phone counseling and mailed printouts, while school-centered programs were conducted across a range of 3 to 36 sessions during after-school hours over two years. Six studies included low-intensity programs (six or fewer sessions), and 16 studies included high-intensity programs (more than seven sessions). Most school-based programs were also high-intensity programs $(\mathrm{k}=14)$ (Table 2$)$.

\section{The Effects of Smoking Prevention Programs on Smok- ing Behavior}

1) Total effect and short-term versus long-term effects of smok-

\section{ing prevention programs on smoking behavior}

Smoking behavior was defined as initiation of smoking, continuation of smoking, and cessation of smoking by active smokers. The total effect size of smoking behavior was calculated to evaluate the effects of 23 smoking prevention programs on smoking behavior. The OR of smoking behavior in all studies was 0.85 (95\% CI=0.77-0.93, $\left.\mathrm{I}^{2}=61.7 \%\right)$, showing a statistically significant effect on reduction of smoking behavior. No publication bias was observed ( $p=.353$ ) (Figure 2-A). Subgroup analysis was conducted based on the measurement time points of programs, and effects were categorized as short-term or long-term. The OR was 0.85 (95\% CI=0.73-1.00, $\left.\mathrm{I}^{2}=0.0 \%\right)$ in 10 studies that reported short-term effects lasting less than 1 year after the program and 0.85 (95\% CI=0.78-0.93, $\left.\mathrm{I}^{2}=68.3 \%\right)$ in the studies that reported long-term effects lasting 
Table 2. Characteristics of the Studies Included in the Meta-analysis $(N=23)$

\begin{tabular}{|c|c|c|c|c|c|c|c|c|c|}
\hline $\begin{array}{l}\text { First } \\
\text { author } \\
\text { (year) }\end{array}$ & Age or grade $(N)$ & $\begin{array}{c}\text { Target } \\
\text { contents }\end{array}$ & $\begin{array}{l}\text { Intervention component } \\
\text { (intensity) }\end{array}$ & Setting & $\begin{array}{l}\text { Background } \\
\text { theory }\end{array}$ & Program operator & Country & Outcome & $\begin{array}{l}\text { Follow-up } \\
\text { measurement } \\
\text { point }\end{array}$ \\
\hline $\begin{array}{l}\text { Bauman } \\
(2001)\end{array}$ & $\begin{array}{l}12-14 \text { years } \\
\text { Exp. }=400 \\
\text { Cont. }=426\end{array}$ & $\mathrm{SP}, \mathrm{AP}$ & $\begin{array}{l}\text { Family-based intervention } \\
\text { ( } 4 \text { sessions): Mailed } 4 \\
\text { booklets, telephone contact }\end{array}$ & OS & $\begin{array}{l}\text { Health belief } \\
\text { model } \\
\text { Value } \\
\text { expectancy } \\
\text { model }\end{array}$ & $\begin{array}{l}\text { Health-related } \\
\text { person (health } \\
\text { educator) }\end{array}$ & USA & SI & 1 year \\
\hline $\begin{array}{l}\text { Ausems } \\
(2002)\end{array}$ & $\begin{array}{l}11.6 \text { years } \\
\text { Exp. } 1=1,002 \\
\text { (in school) } \\
\text { Exp. } 2=871 \\
\text { (out of school) } \\
\text { Exp. } 3=1,068 \\
\text { (in and out of school) } \\
\text { Cont. }=793\end{array}$ & SP & $\begin{array}{l}\text { School-based intervention } \\
\text { (45-60 min/session, } \\
7 \text { sessions) } \\
\text { Out of school intervention: } \\
3 \text { Tailored mail }\end{array}$ & IS, OS & $\begin{array}{l}\text { Social } \\
\text { inoculation } \\
\text { theory } \\
\text { Theory of } \\
\text { reasoned } \\
\text { action } \\
\text { Social cognitive } \\
\text { theory }\end{array}$ & $\begin{array}{l}\text { In-school } \\
\text { intervention: } \\
\text { Trained teacher } \\
\text { Out-of-school } \\
\text { intervention: } \\
\text { Trained educator }\end{array}$ & $\begin{array}{l}\text { Nether- } \\
\text { lands }\end{array}$ & SC, SI & Immediately \\
\hline $\begin{array}{l}\text { Crone } \\
(2003)\end{array}$ & $\begin{array}{l}13 \text { years } \\
\text { Exp. }=1,444 \\
\text { Cont. }=1,118\end{array}$ & SP & $\begin{array}{l}\text { School-based intervention } \\
\text { (3 sessions): } 2 \text { Extra video } \\
\text { lessons, photo expressing }\end{array}$ & IS & No clear report & Trained educator & $\begin{array}{l}\text { Nether- } \\
\text { lands }\end{array}$ & SS, SC, SI & $\begin{array}{l}\text { Immediately, } \\
1 \text { year }\end{array}$ \\
\hline $\begin{array}{l}\text { Curry } \\
(2003)\end{array}$ & $\begin{array}{l}10-12 \text { years } \\
\text { Exp. }=2,020 \\
\text { Cont. }=2,006\end{array}$ & $\mathrm{SP}$ & $\begin{array}{l}\text { Family-based intervention } \\
\text { (3 sessions): } \\
\text { - Children's packet; } \\
\text { Tobacco comics } \\
\text { - Parent; Handbook, } \\
\text { videotape, telephone } \\
\text { counseling, newsletter \& } \\
\text { booster call, physician } \\
\text { message }\end{array}$ & OS & $\begin{array}{l}\text { Based on } \\
\text { previous } \\
\text { research }\end{array}$ & $\begin{array}{l}\text { Health-related } \\
\text { person } \\
\text { (physician): } \\
\text { Telephone } \\
\text { counseling, } \\
\text { booster call }\end{array}$ & USA & SC, SI & $\begin{array}{l}6,12,20 \\
\text { months }\end{array}$ \\
\hline $\begin{array}{l}\text { Ellickson } \\
\text { (2003) }\end{array}$ & $\begin{array}{l}7-8 \text { th grade } \\
\text { Exp. }=2,553 \\
\text { Cont. }=1,723\end{array}$ & SAP & $\begin{array}{l}\text { School-based intervention: } \\
\text { 7th grade (11 sessions), } \\
\text { 8th grade ( } 3 \text { sessions) }\end{array}$ & IS & $\begin{array}{l}\text { Health belief } \\
\text { model } \\
\text { Social learning } \\
\text { theory } \\
\text { Self-efficacy } \\
\text { theory }\end{array}$ & Trained teacher & USA & SC, SI & 18 months \\
\hline $\begin{array}{l}\text { Ausems } \\
(2004)\end{array}$ & $\begin{array}{l}13.1 \text { years } \\
\text { Exp. } 1=525 \\
\text { (in school) } \\
\text { Exp. } 2=513 \\
\text { (out of school) } \\
\text { Exp. } 3=829 \\
\text { (in and out of school) } \\
\text { Cont. }=509\end{array}$ & $\mathrm{SP}$ & $\begin{array}{l}\text { Out-of-school intervention, } \\
\text { computer-based } \\
\text { intervention: } 3 \text { Tailored } \\
\text { letters } \\
\text { In-school-intervention, } \\
\text { school-based intervention } \\
\text { (50 min/session, } 3 \text { sessions) }\end{array}$ & IS, OS & $\begin{array}{l}\text { Social skill } \\
\text {-motivation-de } \\
\text { cision making* }\end{array}$ & $\begin{array}{l}\text { In-school } \\
\text { intervention: } \\
\text { Trained teacher } \\
\text { Out-of-school } \\
\text { intervention: } \\
\text { Trained educator }\end{array}$ & $\begin{array}{l}\text { Nether- } \\
\text { lands }\end{array}$ & $\mathrm{SC}, \mathrm{SI}$ & $\begin{array}{r}6,12,18 \\
\text { months }\end{array}$ \\
\hline $\begin{array}{l}\text { Chou } \\
(2006)\end{array}$ & $\begin{array}{l}12.5 \text { years } \\
\text { Exp. }=1,337 \\
\text { Cont. }=1,324\end{array}$ & $\mathrm{SP}$ & $\begin{array}{l}\text { School-based intervention } \\
\text { (45 min/session/week, } \\
13 \text { sessions): Modified } \\
\text { version of project SMART }\end{array}$ & IS & No clear report & $\begin{array}{l}\text { Health-related } \\
\text { person (public } \\
\text { health official) }\end{array}$ & China & SC, SI & 1 year \\
\hline $\begin{array}{l}\text { Spoth } \\
\text { (2007) }\end{array}$ & $\begin{array}{l}6-7 \text { th grade } \\
\text { Exp. }=5,500 \\
\text { Cont. }=5,281\end{array}$ & SAP & $\begin{array}{l}\text { Out-of-school intervention: } \\
\text { Strengthening families } \\
\text { program ( } 2 \mathrm{hr} \text { / session, } 7 \\
\text { sessions) } \\
\text { In-school intervention: Life } \\
\text { skills training (15 sessions), } \\
\text { Project ALERT (11 sessions) }\end{array}$ & IS, OS & $\begin{array}{l}\text { Resilience } \\
\text { theory } \\
\text { (partnership } \\
\text { model) }\end{array}$ & Trained educator & USA & SC, SI & 18 months \\
\hline $\begin{array}{l}\text { Vartiainen } \\
\text { (2007) }\end{array}$ & $\begin{array}{l}13.8 \text { years } \\
\text { Exp. }=1,244 \\
\text { Cont. }=1,501\end{array}$ & $\mathrm{SP}$ & $\begin{array}{l}\text { School-based intervention } \\
\text { (14 sessions, } 2-3 \text { training } \\
\text { days per year) }\end{array}$ & IS & ASE model* & Trained teacher & Finland & SS, SC, SI & $1,2,3$ years \\
\hline
\end{tabular}

*Reconstructed model based on more than two theories; ${ }^{\dagger}$ Duplicate subject but measurement point is different; ${ }^{\dagger}$ School-based smoking prevention programme; ALERT, adolescent learning experience in resistance training; AP, alcohol prevention; ASE, attitude, social influence, self-efficacy model; Cont., control group; EU-Dap, European drug abuse prevention; Exp., experimental group; IS, in school; OS, out of school; SAP, substance abuse prevention; SC, smoking continuing; SI, smoking initiation; SMART, self-management and resistance training; SP, smoking prevention; SS, stop smoking; TPB, theory of planned behavior. 
Table 2. Characteristics of the Studies Included in the Meta-analysis (Continued) $(N=23)$

\begin{tabular}{|c|c|c|c|c|c|c|c|c|c|}
\hline $\begin{array}{l}\text { First } \\
\text { author } \\
\text { (year) }\end{array}$ & Age or grade $(N)$ & $\begin{array}{c}\text { Target } \\
\text { contents }\end{array}$ & $\begin{array}{l}\text { Intervention component } \\
\text { (intensity) }\end{array}$ & Setting & $\begin{array}{l}\text { Background } \\
\text { theory }\end{array}$ & Program operator & Country & Outcome & $\begin{array}{l}\text { Follow-up } \\
\text { measurement } \\
\text { point }\end{array}$ \\
\hline $\begin{array}{l}\text { Campbell } \\
(2008)\end{array}$ & $\begin{array}{l}12-13 \text { years } \\
\text { Exp. }=5,372 \\
\text { Cont. }=5,358\end{array}$ & SP & $\begin{array}{l}\text { School-based intervention } \\
\text { (10 sessions): A stop } \\
\text { smoking in schools trial } \\
\text { program }\end{array}$ & IS & $\begin{array}{l}\text { Innovation } \\
\text { theory }\end{array}$ & Others (peer led) & England & SC, SI & $\begin{array}{l}\text { Immediately, } \\
1,2 \text { years }\end{array}$ \\
\hline $\begin{array}{l}\text { Faggiano }^{\dagger} \\
(2008, \\
2010)\end{array}$ & $\begin{array}{l}12-14 \text { years } \\
\text { Exp. }=3,098 \\
\text { Cont. }=3,059\end{array}$ & SAP & $\begin{array}{l}\text { School-based intervention } \\
\text { (1 hr/session, } 12 \text { sessions): } \\
\text { - EU-Dap; Knowledge, } \\
\text { attitudes, normative } \\
\text { belief and intrapersonal } \\
\text { skills }\end{array}$ & IS & $\begin{array}{l}\text { Cognitive social } \\
\text { influence } \\
\text { model }^{*}\end{array}$ & Trained educator & Italy & SC, SI & $\begin{array}{l}3 \text { months } \\
(2008) \\
18 \text { months } \\
(2010)\end{array}$ \\
\hline $\begin{array}{l}\text { Ringwalt } \\
(2010)\end{array}$ & $\begin{array}{l}\text { 6th grade } \\
\text { Exp. }=2,765 \\
\text { Cont. }=2,805\end{array}$ & SAP & $\begin{array}{l}\text { School-based intervention } \\
\text { (45 min/session, } \\
11 \text { sessions, } 3 \text { booster } \\
\text { sessions): Project ALERT }\end{array}$ & IS & $\begin{array}{l}\text { Resilience } \\
\text { theory } \\
\text { (partnership } \\
\text { model) }\end{array}$ & Trained teacher & USA & SC, SI & 1 year \\
\hline $\begin{array}{l}\text { Gabrhelik } \\
\text { (2012) }\end{array}$ & $\begin{array}{l}11.4 \text { years } \\
\text { Exp. }=914 \\
\text { Cont. }=839\end{array}$ & SAP & $\begin{array}{l}\text { School-based intervention } \\
\text { (45 min/session, } \\
12 \text { sessions): } \\
\text { - EU-Dap; Knowledge and } \\
\text { attitudes (4 sessions), } \\
\text { interpersonal skills } \\
\text { (4 sessions), and } \\
\text { intrapersonal skills } \\
\text { (4 sessions) }\end{array}$ & IS & $\begin{array}{l}\text { Comprehensive } \\
\text { social } \\
\text { influence } \\
\text { model }^{*}\end{array}$ & Trained teacher & $\begin{array}{l}\text { Czech } \\
\text { Republic }\end{array}$ & SC & $\begin{array}{l}1,3,12,15,24 \\
\text { months }\end{array}$ \\
\hline $\begin{array}{l}\text { Menrath } \\
\text { (2012) }\end{array}$ & $\begin{array}{l}\text { 5-6th grade } \\
\text { Exp. }=446 \\
\text { Cont. }=392\end{array}$ & SAP & $\begin{array}{l}\text { School-based intervention } \\
\text { (13 sessions): Life skills } \\
\text { and self-efficacy program } \\
\text { (no specific mention) }\end{array}$ & IS & $\begin{array}{l}\text { Social influence } \\
\text { approach }\end{array}$ & Trained teacher & Germany & SC & $\begin{array}{l}\text { Immediately, } \\
6 \text { months }\end{array}$ \\
\hline $\begin{array}{l}\text { Luna- } \\
\text { Adame } \\
(2013)\end{array}$ & $\begin{array}{l}11.0 \text { years } \\
\text { Exp. }=482 \\
\text { Cont. }=566\end{array}$ & SP & $\begin{array}{l}\text { School-based intervention } \\
\text { (1st year- } \\
1 \text { hr/session/week, } \\
24 \text { sessions/ 2nd year- } \\
1 \text { hr/session/week, } \\
12 \text { sessions/ } 2 \text { extra } \\
\text { session/each year): } \\
\text { - Life skills training; Role } \\
\text { play, problem-solving, } \\
\text { enhancement of } \\
\text { self-esteem }\end{array}$ & IS & $\begin{array}{l}\text { Social influence } \\
\text { approach }\end{array}$ & $\begin{array}{l}\text { Health-related } \\
\text { person } \\
\text { (psychology } \\
\text { student) }\end{array}$ & Spain & SC, SI & $\begin{array}{l}\text { Immediately, } \\
1 \text { year }\end{array}$ \\
\hline $\begin{array}{l}\text { Malmberg } \\
(2014)\end{array}$ & $\begin{array}{l}13.0 \text { years } \\
\text { Exp. } 1=598 \text { (web) } \\
\text { Exp. } 2=631 \text { (integral) } \\
\text { Cont. }=574\end{array}$ & SAP & $\begin{array}{l}\text { Web-based intervention } \\
\text { (10 sessions): E-learning } \\
\text { Integral intervention: Web + } \\
\text { parental participation, } \\
\text { regulation and monitoring } \\
\text { and counselling }\end{array}$ & Web & ASE model* & $\begin{array}{l}\text { Others (e-learn } \\
\text { tutor) }\end{array}$ & $\begin{array}{l}\text { Nether- } \\
\text { lands }\end{array}$ & SC, SI & 32 months \\
\hline $\begin{array}{l}\text { Andersen } \\
(2015)\end{array}$ & $\begin{array}{l}12.5 \text { years } \\
\text { Exp. }=2,054 \\
\text { Cont. }=1,434\end{array}$ & SP & $\begin{array}{l}\text { School based intervention } \\
\text { (8 sessions a year): } \\
\text { - X: IT intervention }{ }^{\dagger} ; \\
\text { 1) Smoke-free school } \\
\text { grounds, 2) parental } \\
\text { involvement [smoke-free } \\
\text { contract, smoke-free } \\
\text { dialogue], 3) smoke-free } \\
\text { curriculum [self-efficacy] }\end{array}$ & IS & $\begin{array}{l}\text { Social influence } \\
\text { model }\end{array}$ & Trained educator & Denmark & SC & 1 years \\
\hline
\end{tabular}

${ }^{*}$ Reconstructed model based on more than two theories; ${ }^{\dagger}$ Duplicate subject but measurement point is different; ${ }^{\dagger}$ School-based smoking prevention programme; ALERT, adolescent learning experience in resistance training; AP, alcohol prevention; ASE, attitude, social influence, self-efficacy model; Cont., control group; EU-Dap, European drug abuse prevention; Exp., experimental group; IS, in school; OS, out of school; SAP, substance abuse prevention; SC, smoking continuing; SI, smoking initiation; SMART, self-management and resistance training; SP, smoking prevention; SS, stop smoking; TPB, theory of planned behavior. 
Table 2. Characteristics of the Studies Included in the Meta-analysis (Continued) $(N=23)$

\begin{tabular}{|c|c|c|c|c|c|c|c|c|c|}
\hline $\begin{array}{l}\text { First } \\
\text { author } \\
\text { (year) }\end{array}$ & Age or grade $(N)$ & $\begin{array}{c}\text { Target } \\
\text { contents }\end{array}$ & $\begin{array}{l}\text { Intervention component } \\
\text { (intensity) }\end{array}$ & Setting & $\begin{array}{l}\text { Background } \\
\text { theory }\end{array}$ & Program operator & Country & Outcome & $\begin{array}{c}\text { Follow-up } \\
\text { measurement } \\
\text { point }\end{array}$ \\
\hline $\begin{array}{l}\text { Cremers } \\
(2015)\end{array}$ & $\begin{array}{l}10.4 \text { years } \\
\text { Exp. } 1=727 \text { (web) } \\
\text { Exp. } 2=649 \\
\text { (web+text massage) } \\
\text { Cont. }=718\end{array}$ & SP & $\begin{array}{l}\text { Web-based intervention } \\
\text { (6 sessions): Games, } \\
\text { information about } \\
\text { smoking, animated video, } \\
\text { prompt message [text } \\
\text { message and e-mail] }\end{array}$ & Web & I-change model* & $\begin{array}{l}\text { Others (web-based } \\
\text { computer tailored } \\
\text { program) }\end{array}$ & $\begin{array}{l}\text { Nether- } \\
\text { lands }\end{array}$ & SI & 12,25 months \\
\hline Guo (2015) & $\begin{array}{l}13.4 \text { years } \\
\text { Exp. }=1,176 \\
\text { Cont. }=915\end{array}$ & SAP & $\begin{array}{l}\text { School-based intervention } \\
\text { (45 min/session, } \\
10 \text { sessions, } 2 \text { booster } \\
\text { sessions): Integration of } \\
\text { life skills }\end{array}$ & IS & ТPB & Trained educator & Taiwan & $\mathrm{SC}$ & $\begin{array}{l}\text { Immediately, } \\
6,12 \text { months }\end{array}$ \\
\hline $\begin{array}{l}\text { Valdivieso } \\
\text { Lopez } \\
(2015)\end{array}$ & $\begin{array}{l}10.5 \text { years } \\
\text { Exp. }=779 \\
\text { Cont. }=804\end{array}$ & SP & $\begin{array}{l}\text { School-based intervention } \\
(1 \mathrm{hr} / \text { session, } 9 \text { sessions, } \\
7 \text { modules, } 2 \text { per course } \\
\text { level, over } 3 \text { years): } \\
\text { Activities, workshop, class } \\
\text { sessions }\end{array}$ & IS & $\begin{array}{l}\text { Social influence } \\
\text { model }\end{array}$ & $\begin{array}{l}\text { Health-related } \\
\text { person (school } \\
\text { nurse, primary } \\
\text { care nurse) }\end{array}$ & Spain & SI & 4 years \\
\hline $\begin{array}{l}\text { Baldus } \\
\text { (2016) }\end{array}$ & $\begin{array}{l}12-13 \text { years } \\
\text { Exp. }=147 \\
\text { Cont. }=145\end{array}$ & SAP & $\begin{array}{l}\text { Family-based intervention } \\
\text { (7 sessions, weekly and } \\
4 \text { booster sessions): } \\
\text { 1) Youth module } \\
\text { [self-efficacy, coping with } \\
\text { stress and peer pressure], } \\
\text { 2) parent module } \\
\text { [parenting style], } \\
\text { 3) ensuing family session } \\
\text { [communication pattern] }\end{array}$ & OS & $\begin{array}{l}\text { Socio-ecological } \\
\text { model }\end{array}$ & Trained educator & Germany & SC, SI & $\begin{array}{l}\text { Immediately, } \\
6,18 \text { months }\end{array}$ \\
\hline $\begin{array}{l}\text { Krist } \\
\text { (2016) }\end{array}$ & $\begin{array}{l}13 \pm 0.6 \text { years } \\
\text { Exp. } 1=1,142 \\
\text { (student only) } \\
\text { Exp. } 2=980 \\
\text { (student-parent) } \\
\text { Cont. }=679\end{array}$ & SP & $\begin{array}{l}\text { School-based intervention: } \\
\text { - Student-only intervention } \\
\text { ( } 2 \text { hr/session, } 1 \text { session); } \\
\text { Information and skills, } \\
\text { harmful effects of } \\
\text { cigarettes } \\
\text { - Student-parent } \\
\text { intervention (1 session); } \\
\text { Student-only intervention } \\
\text { + health coaching + } \\
\text { newsletter } 8 \text { months after } \\
\text { taught }\end{array}$ & IS & No clear report & Trained educator & Germany & SC, SI & 1,2 years \\
\hline $\begin{array}{r}\text { Brinker } \\
(2017)\end{array}$ & $\begin{array}{l}12.4 \text { years } \\
\text { Exp. }=318 \\
\text { Cont. }=401\end{array}$ & SP & $\begin{array}{l}\text { School-based intervention } \\
\text { (60 min/session, } \\
2 \text { sessions): Photo-aging } \\
\text { desktop program } \\
\text { [application and computer] }\end{array}$ & IS & No clear report & $\begin{array}{l}\text { Health-related } \\
\text { person (medical } \\
\text { student) }\end{array}$ & Germany & SS, SC, SI & 1 year \\
\hline
\end{tabular}

*Reconstructed model based on more than two theories; ${ }^{\dagger}$ Duplicate subject but measurement point is different; ${ }^{\dagger}$ School-based smoking prevention programme; ALERT, adolescent learning experience in resistance training; AP, alcohol prevention; ASE, attitude, social influence, self-efficacy model; Cont., control group; EU-Dap, European drug abuse prevention; Exp., experimental group; IS, in school; OS, out of school; SAP, substance abuse prevention; SC, smoking continuing; SI, smoking initiation; SMART, self-management and resistance training; SP, smoking prevention; SS, stop smoking; TPB, theory of planned behavior.

at least 1 year. No publication bias was observed in the studies that analyzed short-term and long-term effects $(p=.766, p=$ .297, respectively) (Figure 2-B).

\section{2) The effects of smoking prevention programs by program type, provider, setting, and intensity}

(1) Effect size according to program type

The types of smoking prevention programs were family- centered, school-based, and web-based. No publication bias was observed in family-based and school-based programs $(p=$ $.294, p=.561$, respectively). For web-based programs, calculations were not possible since they were only included in two studies. The effect of smoking behavior across the 18 schoolbased programs was an OR of $0.79\left(95 \% \mathrm{CI}=0.75-0.83, \mathrm{I}^{2}=\right.$ $52.1 \%)$. The effects were not significant for family-centered programs $(k=3)$ or web-based programs $(k=2)$ (Figure 2-C). 

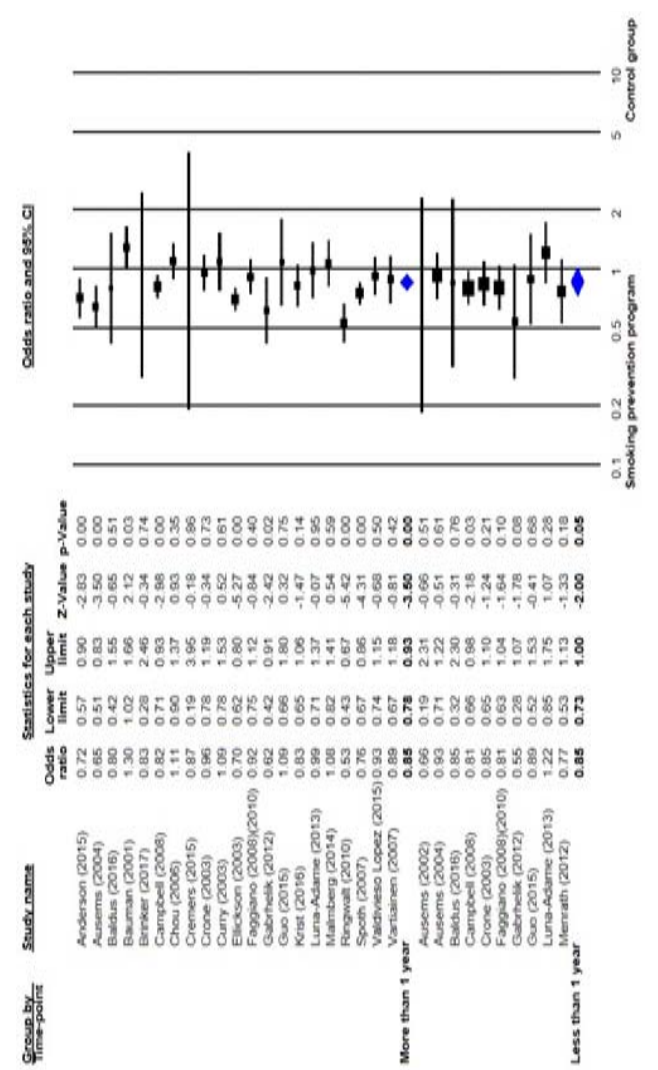

(a)

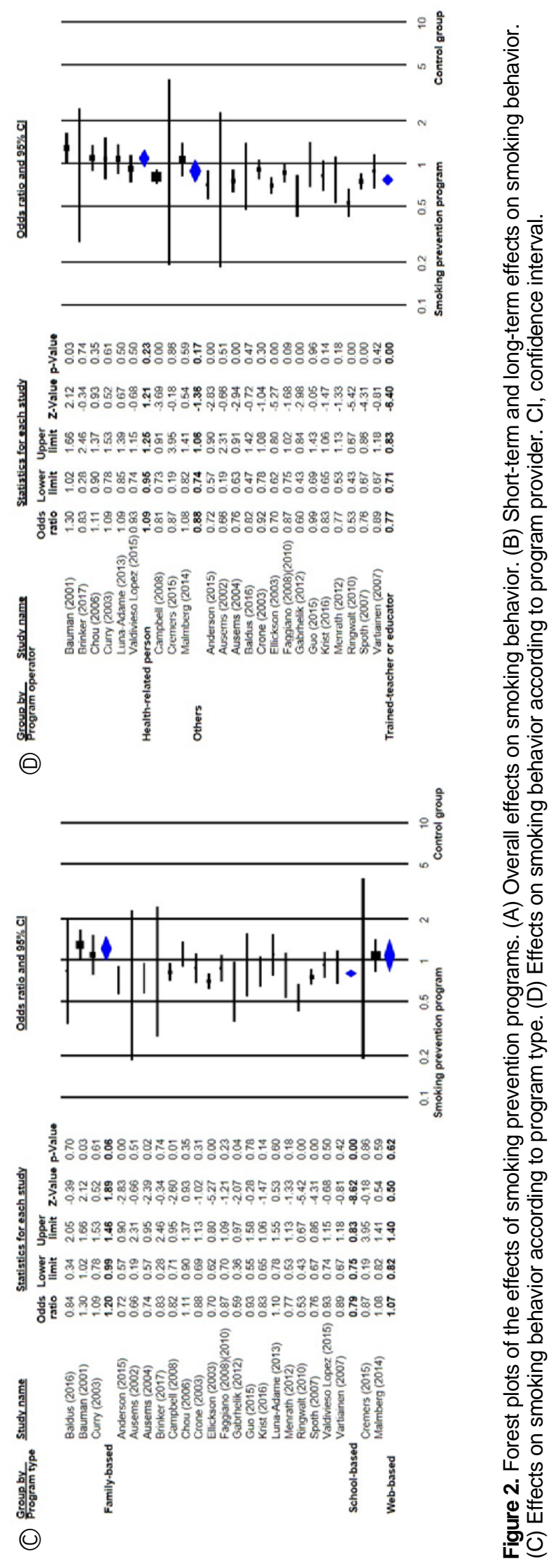




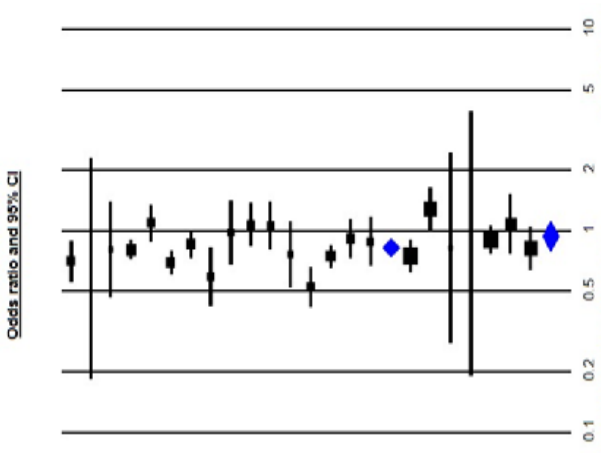

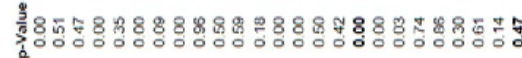

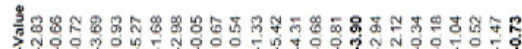

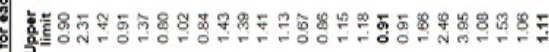

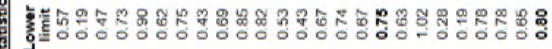

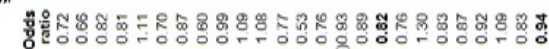
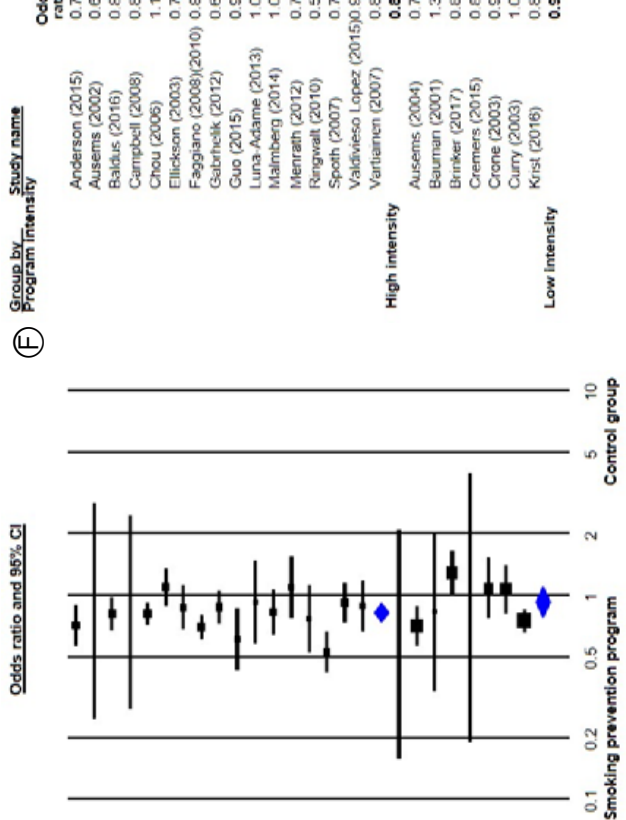

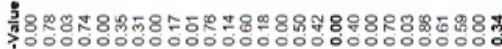
畜 3ำ 势

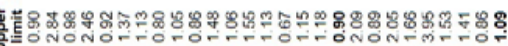

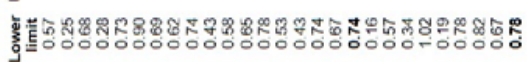

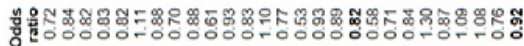

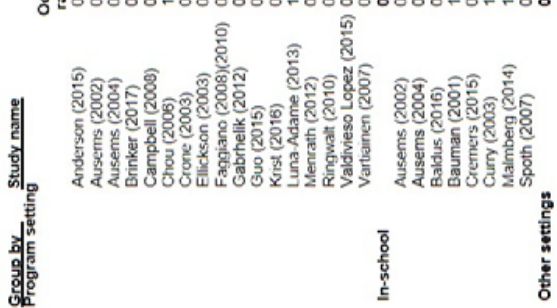

(1)
(2) Effect size according to program provider

The types of program providers were trained teachers or educators, health-related persons, or others. No publication bias was observed in each analysis $(p=.812, p=.773, p=.612$, respectively). The effect of smoking behavior was an OR of $0.77\left(95 \% \mathrm{CI}=0.71-0.83, \mathrm{I}^{2}=0.0 \%\right)$ in the 14 studies in which trained teachers or educators conducted the program. The effect was statistically insignificant for programs conducted by health-related persons or others (Figure 2-D).

(3) Effect size according to program setting

The types of settings in which smoking prevention programs took place were either in-school settings or other. No publication bias was observed in each analysis $(p=.551, p=$ .558 , respectively). The effect of smoking behavior was an OR of $0.82\left(95 \% \mathrm{CI}=0.74-0.90, \mathrm{I}^{2}=53.1 \%\right)$ across the 18 studies in which smoking prevention programs were conducted in a school environment. The effect of smoking behavior in programs conducted in combined settings was not statistically significant (Figure 2-E).

(4) Effect size according to program intensity

Programs were considered either high-intensity or low-intensity. No publication bias was observed in each analysis ( $p=.613, p=.742$, respectively). The effect of smoking behavior was an OR of 0.82 (95\% CI=0.75- $\left.0.91, \mathrm{I}^{2}=66.6 \%\right)$ across the 16 studies in which smoking prevention programs were considered high intensity. However, the effect of programs with low intensity was not significant (Figure 2-F).

\section{DISCUSSION}

Smoking prevention for young adolescents is important because young adolescents who smoke are more likely to continue smoking through mid-to-late adolescence and into adulthood, negatively affecting their future health [1,2]. Various smoking prevention programs have been conducted to discourage smoking behavior in young adolescents with mixed results. This study aimed to objectively examine the usefulness of smoking prevention programs targeted to young adolescents and propose potential directions for developing smoking prevention programs tailored to the unique developmental characteristics of young adolescents.

The intervention programs conducted in the included studies were either developed for smoking behavior or adopted from smoking and substance abuse programs with the intention to control smoking behaviors. Smoking prevention programs were effective at controlling smoking both in the short and long term. Subgroup analysis was conducted to compare studies by program setting. School-based smoking prevention 
programs were used in 18 studies, with an OR of 0.79 . Thomas, McLellan, and Perera [26] analyzed a school-based smoking prevention program for children and adolescents between 8 and 18 years old, which showed significant short-term and long-term effects. In this study, most school-based programs were based on social influence theory or theories of motivation, often including high-intensity social skill and interpersonal skill training, methods for improving self-efficacy, and skills for coping with the urge to smoke. In a study by Thomas et al. [26], intervention programs based on social influence theory and social competence were effective at reducing smoking behavior compared to other multimodal approaches. A smoking prevention program based on social influence theory focuses on the social factors of smoking behavior and teaches (1) how to say no to smoking, (2) how to cope with peer pressure, and (3) how to avoid high-risk situations that encourage or entail smoking. Programs based on social competency, which focus on adolescents' social skills, can help teenagers refuse smoking proposals by improving general social competence and personal and social skills [26]. The specific strategies outlined in these theories can be applied to intervention programs for young adolescents in highrisk environments for smoking and can be considered when designing future school-based programs.

Our meta-analysis found no significant effects on smoking prevention resulting from family-centered or web-based programs. However, a systematic review by Thomas, Baker, Thomas, and Lorenzetti [27] showed that a family-based smoking prevention program for individuals between 5 and 18 years old had significant effects (relative risk=0.76) when conducted for families or in groups in a home setting with consistent prompts and contact. Their study suggested that family-centered programs can be effective when authoritative parenting style training is included in the program [27]. Young adolescents are often sensitive to and affected by their families since they are still in a period when parent-child relationships are re-established [28]. However, this result should be interpreted with caution, since only a small number of these studies were included in the analysis. Further studies should be conducted to evaluate the effects of high-intensity family-centered programs that also include parenting behavior.

The effect of web-based smoking prevention programs on smoking behavior was not significant in our study. This result corresponds to the findings of a previous study that conducted a meta-analysis of the literature on web-based smoking cessation programs, showing that web-based programs were effective at reducing smoking behavior for adults, but not for adolescents [29]. Web-based smoking prevention programs that reflect the developmental characteristics of young adolescents can be developed and conducted in the future, however, as the number of studies on web-based programs continues to increase.

In this study, smoking prevention programs were most effective when conducted by a trained teacher. A close bond between a young adolescent and his or her teacher is considered an important protective factor preventing juvenile delinquency and strengthening an adolescent's ability to overcome problematic behavior [30]. Therefore, school-based programs conducted by teachers with whom students may have a close bond can have positive effects on the prevention or reduction of smoking behavior among students. On the contrary, the effect of programs was not significant when conducted by health specialists or students studying health-related majors. Only three such studies were included in the analysis, however, which is fairly low. Consequently, the effect of program providers on smoking prevention should be further investigated by controlling for potential confounding factors such as program intensity.

Program setting can also be a critical factor affecting smoking behavior. The greatest effects on smoking behavior were seen in programs conducted in school settings. Programs conducted in combined settings or via websites were not as effective, which may also be related to program providers not being trained teachers for programs outside of school settings. When programs were provided at school settings, participants likely had support from teachers or friends [30], which may have led to better outcomes for young adolescents.

The OR was 0.81 for high-intensity programs, which consisted of seven or more sessions. No effect was observed for low-intensity programs. The findings of previous meta-analyses showed mixed results. Sussman et al. [15] found no association between the number of sessions and the smoking cessation rate. However, Kim et al. [10] found that a reduction in the smoking rate was more likely when a program met or exceeded the average number of sessions (6.5 times) and had sufficiently long sessions (at least 90 minutes). Programs in this study were considered high-intensity when they were conducted for seven or more sessions with each session lasting between 45 and 60 minutes. High-intensity programs can reduce smoking behavior by discouraging smoking initiation and continuation of smoking behavior, and encouraging cessation.

Results from this meta-analysis suggest that the future direction for developing and conducting smoking prevention programs tailored to young adolescents in South Korea should be to create high-intensity school-centered programs conducted by trained teachers. However, no RCTs have yet investigated this issue in South Korea, meaning that the current state of smoking prevention programs among early adolescents in South Korea was not reflected. Particular caution is needed for interpreting the results, as $\mathrm{I}^{2}$ was $51.3 \%-68.3 \%$ of the 
included studies, reflecting considerable heterogeneity. In addition, the outcome variable of this study was smoking-related behaviors, mostly assessed using self-reported results on the initiation, continuation, and cessation of smoking, and not objective indicators (e.g., such as urinary nicotine levels) or psychological factors (e.g., such as willingness to quit smoking, self-efficacy, and reasons for smoking). In addition, this analysis included adolescents who did not have smoking experience, adolescents with past smoking experience, and adolescents who actively smoked. Therefore, interventions should be conducted with particular caution when applied to all young adolescents. Based on the findings and limitations of this study, further research targeting children and young adolescents in South Korea is needed with an RCT design, incorporating both self-reported smoking behaviors as well as physical examination items in the outcome variables, such as urinary nicotine levels, in order to obtain more objective results.

\section{CONCLUSION}

This systematic review and meta-analysis aimed to objectively analyze the effects of smoking prevention programs on improving smoking behavior among young adolescents and to further propose objective grounds for developing or implementing effective smoking prevention programs tailored to young adolescents. The results of a meta-analysis of 23 studies with RCTs showed that smoking prevention programs reduced the smoking behavior of young adolescents. High-intensity school-based programs conducted by trained instructors were especially effective. However, the effect sizes were small and the studies included in the analysis were heterogeneous. Therefore, caution is warranted in interpreting and applying the results. Additional research, particularly with an RCT design, is required to explore the effect of family-centered and web-based programs.

\section{ORCID}

Rhayun Song https://orcid.org/0000-0001-9461-4246

Moonkyoung Park https://orcid.org/0000-0001-6693-0540

\section{Authors' contribution}

Conceptualization, Methodology: all authors; Data curation, Formal analysis: all authors; Project administration and Supervision: all authors; Writing-original draft, Writing-review and editing: all authors; Final approval of published version: all authors.

\section{Conflict of interest}

No existing or potential conflict of interest relevant to this article was reported.

\section{Funding}

This study was supported by the research fund of Chungnam National University in 2018.

\section{Data availability}

Please contact the corresponding author for data availability.

\section{Acknowledgements}

None.

\section{REFERENCES}

1. Strand TE, Malayeri C, Eskonsipo PKJ, Grimsrud TK, Norstein J, Grotmol T. Adolescent smoking and trends in lung cancer incidence among young adults in Norway 1954-1998. Cancer Causes and Control. 2004;15(1):27-33.

https://doi.org/10.1023/B:CACO.0000016575.31651.b0

2. Kim YE, Kim DH, Roh YK, Ju SY, Yoon YJ, Nam GE, et al. Relationship between serum ferritin levels and dyslipidemia in Korean adolescents. PLoS One. 2016;11(4):e0153167. https://doi.org/10.1371/journal.pone.0153167

3. Curry SJ, Mermelstein RJ, Sporer AK. Therapy for specific problems: Youth tobacco cessation. Annual Review of Psychology. 2009; 60:229-255.

https://doi.org/10.1146/annurev.psych.60.110707.163659

4. Korea Disease Control and Prevention Agency. The fifteenth Korea youth risk behavior web-based survey in 2019 [Internet]. Cheongju: Korea Disease Control and Prevention Agency; 2019 [cited 2020 December 21]. Available from: https://www.kdca.go.kr/yhs/

5. Casey BJ, Getz S, Galvan A. The adolescent brain. Developmental Review. 2008;28(1):62-77.

https://doi.org/10.1016/j.dr.2007.08.003

6. Petrescu DC, Vasiljevic M, Pepper JK, Ribisl KM, Marteau TM. What is the impact of e-cigarette adverts on children's perceptions of tobacco smoking? An experimental study. Tobacco Control. 2017;26(4):421-427.

https://doi.org/10.1136/tobaccocontrol-2016-052940

7. Fergusson DM, Horwood LJ. Transitions to cigarette smoking during adolescence. Addictive Behaviors. 1995;20(5):627-642.

https://doi.org/10.1016/0306-4603(95)00023-6

8. Lee JS, Kim H. Meta-analysis of effectiveness of smoking preven- 
tion program for elementary school students. Korean Journal of Health Education and Promotion. 2017;34(4):99-110.

https://doi.org/10.14367/kjhep.2017.34.4.99

9. Fanshawe TR, Halliwell W, Lindson N, Aveyard P, LivingstoneBanks J, Hartmann-Boyce J. Tobacco cessation interventions for young people. The Cochrane Database of Systematic Reviews. 2017;11(11):CD003289.

https://doi.org/10.1002/14651858.CD003289.pub6

10. Kim Y, Park I, Park JS. Meta-analysis of effects on adolescent smoking cessation programs in Korea. Journal of Korean Academy of Nursing. 2008;38(2):204-216.

11. Corepal R, Tully MA, Kee F, Miller SJ, Hunter RF. Behavioural incentive interventions for health behaviour change in young people (5-18 years old): A systematic review and meta-analysis. Preventive Medicine. 2018;110:55-66.

https://doi.org/10.1016/j.ypmed.2018.02.004

12. De Kleijn MJJ, Farmer MM, Booth M, Motala A, Smith A, Sherman $\mathrm{S}$, et al. Systematic review of school-based interventions to prevent smoking for girls. Systematic Reviews. 2015;4:109.

https://doi.org/10.1186/s13643-015-0082-7

13. Carson KV, Brinn MP, Labiszewski NA, Esterman AJ, Chang AB, Smith BJ. Community interventions for preventing smoking in young people. The Cochrane Database of Systematic Reviews. 2011;7: CD001291. https://doi.org/10.1002/14651858.CD001291.pub2

14. Park E, Drake E. Systematic review: Internet-based program for youth smoking prevention and cessation. Journal of Nursing Scholarship. 2015;47(1):43-50. https://doi.org/10.1111/jnu.12104

15. Sussman S, Sun P, Dent CW. A meta-analysis of teen cigarette smoking cessation. Health Psychology. 2006;25(5):549-557. https://doi.org/10.1037/0278-6133.25.5.549

16. Brinker TJ, Owczarek AD, Seeger W, Groneberg DA, Brieske CM, Jansen $\mathrm{P}$, et al. A medical student-delivered smoking prevention program, education against tobacco, for secondary schools in Germany: Randomized controlled trial. Journal of Medical Internet Research. 2017;19(6):e199.

https://doi.org/10.2196/jmir.7906

17. Valdivieso Lopez E, Rey-Reñones C, Rodriguez-Blanco T, Ferre Grau C, Arija V, Barrera Uriarte ML, et al. Efficacy of a smoking prevention programme in Catalan secondary schools: A cluster-randomized controlled trial in Spain. Addiction. 2015;110(5):852-860.

https://doi.org/10.1111/add.12833

18. Bast LS, Due P, Bendtsen P, Ringgard L, Wohllebe L, Damsgaard MT, et al. High impact of implementation on school-based smoking prevention: The X: IT study-a cluster-randomized smoking prevention trial. Implementation Science. 2016;11(1):125. https://doi.org/10.1186/s13012-016-0490-7

19. Baldus C, Thomsen M, Sack PM, Bröning S, Arnaud N, Daubmann A, et al. Evaluation of a German version of the Strengthening Families
Programme 10-14: A randomised controlled trial. European Journal of Public Health. 2016;26(6):953-959.

https://doi.org/10.1093/eurpub/ckw082

20. Moher D, Liberati A, Tetzlaff J, Altman DG. Preferred reporting items for systematic reviews and meta-analyses: The PRISMA statement. PLoS Medicine. 2009;6(7):e1000097. https://doi.org/10.1371/journal.pmed.1000097

21. Bornstein MH, Hahn CS, Haynes OM. Social competence, externalizing, and internalizing behavioral adjustment from early childhood through early adolescence: Developmental cascades. Development and Psychopathology. 2010;22(4):717-735.

https://doi.org/10.1017/S0954579410000416

22. Scottish Intercollegate Guidelines Network. SIGN 50: A guideline developer's handbook [Internet]. Edinburgh: Scottish Intercollegate Guidelines Network; 2019 [cited 2019 November 30]. Available from:

https://www.sign.ac.uk/our-guidelines/sign-50-a-guideline-de velopers-handbook/

23. Higgins J, Thomas J, Chandler J, Cumpston M, Li T, Page M, et al. Cochrane handbook for systematic reviews of interventions. 2nd ed. New Jersey: Wiley-Blackwell; 2019. p. 362-588.

24. Egger M, Davey Smith G, Schneider M, Minder C. Bias in metaanalysis detected by a simple, graphical test. BMJ. 1997;315(7109): 629-634. https://doi.org/10.1136/bmj.315.7109.629

25. Borenstein M. Common mistakes in meta-analysis and how to avoid them. New Jersey: Biostat Inc.; 2019. p. 13-41.

26. Thomas RE, McLellan J, Perera R. Effectiveness of school-based smoking prevention curricula: Systematic review and meta-analysis. BMJ Open. 2015;5(3):e006976. https://doi.org/10.1136/bmjopen-2014-006976

27. Thomas RE, Baker PRA, Thomas BC, Lorenzetti DL. Family-based programmes for preventing smoking by children and adolescents. The Cochrane Database of Systematic Reviews. 2015;2:CD004493. https://doi.org/10.1002/14651858.CD004493.pub3

28. Agerup T, Lydersen S, Wallander J, Sund AM. Associations between parental attachment and course of depression between adolescence and young adulthood. Child Psychiatry and Human Development. 2015;46(4):632-642.

https://doi.org/10.1007/s10578-014-0506-y

29. Myung SK, McDonnell DD, Kazinets G, Seo HG, Moskowitz JM. Effects of web- and computer-based smoking cessation programs: Meta-analysis of randomized controlled trials. Archives of Internal Medicine. 2009;169(10):929-937. https://doi.org/10.1001/archinternmed.2009.109

30. Hallinan MT. Teacher influences on students' attachment to school. Sociology of Education. 2008;81(3):271-283. https://doi.org/10.1177/003804070808100303 


\section{Appendix 1. Citations for Studies Included in this Study}

1. Bauman KE, Foshee VA, Ennett ST, Pemberton M, Hicks KA, King $\mathrm{TS}$, et al. The influence of a family program on adolescent tobacco and alcohol use. American Journal of Public Health. 2001;91(4): 604-610. https://doi.org/10.2105/ajph.91.4.604

2. Ausems M, Mesters I, Van Breukelen G, De Vries H. Short-term effects of a randomized computer-based out-of-school smoking prevention trial aimed at elementary schoolchildren. Preventive Medicine. 2002;34(6):581-589.

https://doi.org/10.1006/pmed.2002.1021

3. Curry SJ, Hollis J, Bush T, Polen M, Ludman EJ, Grothaus L, et al. A randomized trial of a family-based smoking prevention intervention in managed care. Preventive Medicine. 2003;37(6):617-626. https://doi.org/10.1016/j.ypmed.2003.09.015

4. Crone M, Reijneveld SA, Willemsen MC, Van Leerdam FJ, Spruijt RD, Hira Sing RA. Prevention of smoking in adolescents with lower education: A school based intervention study. Journal of Epidemiology and Community Health. 2003;57(9):675-680. https://doi.org/10.1136/jech.57.9.675

5. Ellickson PL, McCaffrey DF, Ghosh-Dastidar B, Longshore DL. New inroads in preventing adolescent drug use: Results from a large-scale trial of Project ALERT in middle schools. American Journal of Public Health. 2003;93(11):1830-1836. https://doi.org/10.2105/ajph.93.11.1830

6. Ausems M, Mesters I, Van Breukelen G, De Vries H. Effects of inschool and tailored out-of-school smoking prevention among Dutch vocational school students. Health Education Research. 2004;19(1):51-63. https://doi.org/10.1093/her/cyg001

7. Chou CP, Li Y, Unger JB, Xia J, Sun P, Guo Q, et al. A randomized intervention of smoking for adolescents in urban Wuhan, China. Preventive Medicine. 2006;42(4):280-285.

https://doi.org/10.1016/j.ypmed.2006.01.002

8. Spoth R, Redmond C, Shin C, Greenberg M, Clair S, Feinberg M. Substance-use outcomes at 18 months past baseline: The PROSPER community-university partnership trial. American Journal of Preventive Medicine. 2007;32(5):395-402.

https://doi.org/10.1016/j.amepre.2007.01.014

9. Vartiainen E, Pennanen M, Haukkala A, Dijk F, Lehtovuori R, De Vries $\mathrm{H}$. The effects of a three-year smoking prevention programme in secondary schools in Helsinki. European Journal of Public Health. 2007;17(3):249-256.

https://doi.org/10.1093/eurpub/ckl107

10. Campbell R, Starkey F, Holliday J, Audrey S, Bloor M, ParryLangdon N, et al. An informal school-based peer-led intervention for smoking prevention in adolescence (ASSIST): A cluster randomised trial. The Lancet. 2008;371(9624):1595-1602.

https://doi.org/10.1016/S0140-6736(08)60692-3

11. Faggiano F, Galanti MR, Bohrn K, Burkhart G, Vigna-Taglianti F, Cuomo L, et al. The effectiveness of a school-based substance abuse prevention program: EU-Dap cluster randomised controlled trial. Preventive Medicine. 2008;47(5):537-543.

https://doi.org/10.1016/j.ypmed.2008.06.018

12. Faggiano F, Vigna-Taglianti F, Burkhart G, Bohrn K, Cuomo L, Gregori D, et al. The effectiveness of a school-based substance abuse prevention program: 18-Month follow-up of the EU-Dap cluster randomized controlled trial. Drug and Alcohol Dependence. 2010;108(1-2):56-64.

https://doi.org/10.1016/j.drugalcdep.2009.11.018

13. Ringwalt CL, Clark HK, Hanley S, Shamblen SR, Flewelling RL. The effects of Project ALERT one year past curriculum completion. Prevention Science. 2010;11(2):172-184. https://doi.org/10.1007/s11121-009-0163-8

14. Gabrhelik R, Duncan A, Miovsky M, Furr-Holden CDM, Stastna L, Jurystova L. "Unplugged": A school-based randomized control trial to prevent and reduce adolescent substance use in the Czech Republic. Drug and Alcohol Dependence. 2012;124(1-2):79-87. https://doi.org/10.1016/j.drugalcdep.2011.12.010

15. Menrath I, Mueller-Godeffroy E, Pruessmann C, Ravens-Sieberer U, Ottova V, Pruessmann M, et al. Evaluation of school-based life skills programmes in a high-risk sample: A controlled longitudinal multi-centre study. Journal of Public Health. 2012;20(2):159-170. https://doi.org/10.1007/s10389-011-0468-5

16. Luna-Adame M, Carrasco-Giménez TJ, Rueda-García MDM. Evaluation of the effectiveness of a smoking prevention program based on the 'Life Skills Training' approach. Health Education Research. 2013;28(4):673-682.

https://doi.org/10.1093/her/cyt061

17. Malmberg M, Kleinjan M, Overbeek G, Vermulst A, Monshouwer $\mathrm{K}$, Lammers J, et al. Effectiveness of the 'Healthy School and Drugs' prevention programme on adolescents' substance use: A randomized clustered trial. Addiction. 2014;109(6):1031-1040. https://doi.org/10.1111/add.12526

18. Andersen A, Krølner R, Bast LS, Thygesen LC, Due P. Effects of the $\mathrm{X}$ : IT smoking intervention: A school-based cluster randomized trial. International Journal of Epidemiology. 2015;44(6):1900-1908. https://doi.org/10.1093/ije/dyv145

19. Cremers HP, Mercken L, Candel M, De Vries H, Oenema A. A web-based, computer-tailored smoking prevention program to prevent children from starting to smoke after transferring to secondary school: Randomized controlled trial. Journal of Medical Internet Research. 2015;17(3):e59.

https://doi.org/10.2196/jmir.3794

20. Guo JL, Lee TC, Liao JY, Huang CM. Prevention of illicit drug use through a school-based program: Results of a longitudinal, cluster-randomized controlled trial. The Journal of Adolescent Health. 2015;56(3):314-322.

https://doi.org/10.1016/j.jadohealth.2014.12.003 
21. Valdivieso López E, Rey-Reñones C, Rodriguez-Blanco T, Ferre Grau C, Arija V, Barrera Uriarte ML, et al. Efficacy of a smoking prevention programme in Catalan secondary schools: A cluster-randomized controlled trial in Spain. Addiction. 2015;110(5):852-860. https://doi.org/10.1111/add.12833

22. Baldus C, Thomsen M, Sack PM, Bröning S, Arnaud N, Daubmann A, et al. Evaluation of a German version of the Strengthening Families Programme 10-14: A randomised controlled trial. European Journal of Public Health. 2016;26(6):953-959. https://doi.org/10.1093/eurpub/ckw082

23. Krist L, Lotz F, Bürger C, Ströbele-Benschop N, Roll S, Rieckmann
$\mathrm{N}$, et al. Long term effectiveness of a combined student-parent and a student-only smoking prevention intervention among 7 th grade school children in Berlin, Germany. Addiction. 2016;111(12): 2219-2229. https://doi.org/10.1111/add.13537

24. Brinker TJ, Owczarek AD, Seeger W, Groneberg DA, Brieske CM, Jansen $\mathrm{P}$, et al. A medical student-delivered smoking prevention program, education against tobacco, for secondary schools in Germany: Randomized controlled trial. Journal of Medical Internet Research. 2017;19(6):e199.

https://doi.org/10.2196/jmir.7906 